\title{
Dynamic interaction between elastic plate and transversely isotropic poroelastic medium
}

\author{
Suraparb Keawsawasvong, and Teerapong Senjuntichai* \\ Applied Mechanics and Structures Research Unit, Department of Civil Engineering, Faculty of Engineering, Chulalongkorn University, \\ Bangkok, 10330, Thailand.
}

\begin{abstract}
In this paper, dynamic response of an elastic circular plate, under axisymmetric time-harmonic vertical loading, resting on a transversely isotropic poroelastic half-space is investigated. The plate-halfspace contact surface is assumed to be smooth and fully permeable. The discretization techniques are employed to solve the unknown normal traction at the contact surface based on the solution of flexibility equations. The vertical displacement of the plate is represented by an admissible function containing a set of generalized coordinates. Solutions for generalized coordinates are obtained by establishing the equation of motion of the plate through the application of Lagrange's equations of motion. Selected numerical results corresponding to the deflections of a circular plate, with different degrees of flexibility, resting on a transversely isotropic poroelastic half-space are presented.
\end{abstract}

\section{Introduction}

Biot [1] proposed a theory of wave propagations in a poroelastic material, which is a two-phase material consisting of an elastic solid with voids filled with water. Thereafter, Biot's poroelastodynamics theory has been employed by many researchers to study dynamic interaction problems between foundations and fluidsaturated porous media due to its close relevance to various practical problems in civil engineering. For example, the dynamic responses of rigid foundations under vertical loading were investigated by Kassir and $\mathrm{Xu}$ [2] for rigid strips, Jin and Liu [3], Zeng and Rajapakse [4] and Ai et al. [5] for rigid circular plates, and Halpern and Christiano [6], Senjuntichai et al. [7-8] and Keawsawasvong and Senjuntichai [9] for rigid rectangular plates. In addition, the influence of the flexibility of foundations on dynamic interaction between foundations and supporting poroelastic media was also investigated by Senjuntichai and Kaewjuea [10] for multiple flexible strips and Senjuntichai and Sapsathiarn [11] for a flexible circular plate.

Mechanical behaviours of natural soils and rocks commonly exhibit certain degrees of anisotropy owing to deposition or sedimentation processes. As a result, the material properties of such materials in vertical and horizontal directions are generally different. Constitutive relations for anisotropic poroelastic materials were introduced by Biot [12] to capture the anisotropy effect of the two-phase materials. Biot [13] then proposed governing equations for wave propagations in anisotropic poroelastic materials. The interpretation of material properties of transversely isotropic poroelastic media was later presented by Cheng [14] in order to illustrate the relations between the results obtained from the laboratory measurement and the parameters in the transversely isotropic poroelastic materials proposed by Biot [13]. By adopting Biot's equations [13], Kumar et al. [15], Sahebkar and Eskandari-Ghadi [16] and Pooladi et al. [17] presented solutions of a transversely isotropic poroelastic medium subjected to time harmonic point or uniform loading. A review of literature reveals that a soil-structure interaction problem involving a flexible plate and a transversely isotropic medium has never been appeared in the past.

This paper presents vertical vibrations of an elastic circular plate of radius $a$ under axisymmetric timeharmonic vertical loading resting on a transversely isotropic poroelastic half-space as shown in Fig. 1. The plate-half-space contact surface is assumed to be smooth and fully permeable. A classical thin-plate theory [18] is adopted for the circular plate, and the plate deflection is represented by an admissible function containing a set of generalized coordinates. A representation for contact traction is established in terms of generalized coordinates through the solution of a flexibility equation based on a fundamental solution of the half-space based on Biot's theory [13]. The equation of motion of the plate in terms of generalized coordinates are established through the application of Lagrange's equation of motion. The accuracy of the present solution is verified by comparisons with existing solutions. Selected

\footnotetext{
* Corresponding author: Teerapong.S@chula.ac.th
} 
numerical results are presented to portray the influence of relative flexibility and the frequency of excitation on the deflection of the circular plate.

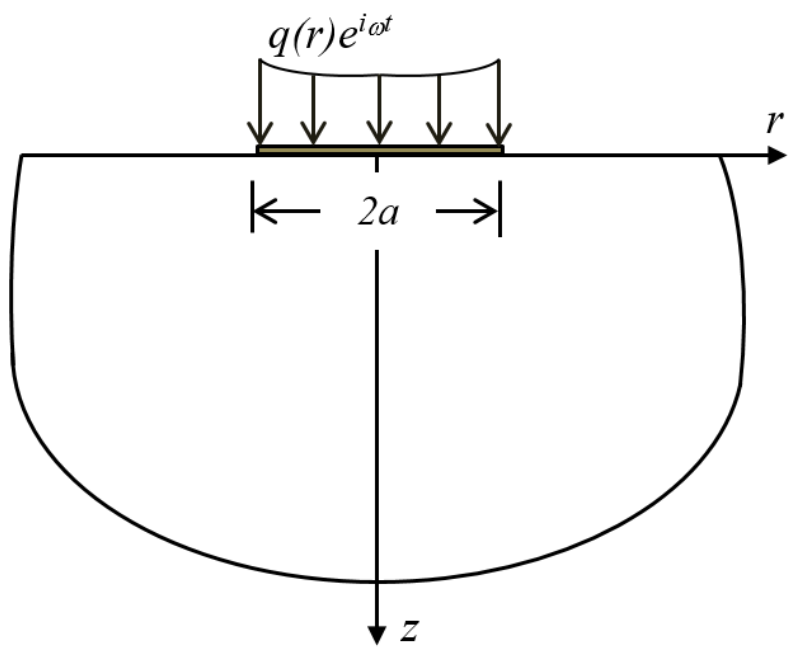

Fig. 1. A circular plate resting on a transversely isotropic poroelastic half-space.

\section{Basic equations and influence functions}

Consider a transversely isotropic poroelastic medium undergoing axisymmetric deformations with the cylindrical coordinate system $(r, \theta, z)$ as shown in Fig. 1. According to Cheng [14], the constitutive relations for transversely isotropic poroelastic materials can be expressed for axisymmetric deformations as,

$$
\begin{aligned}
& \sigma_{r r}=c_{11} \frac{\partial u_{r}}{\partial r}+c_{12} \frac{u_{r}}{r}+c_{13} \frac{\partial u_{z}}{\partial z}-\alpha_{h} p \\
& \sigma_{\theta \theta}=c_{12} \frac{\partial u_{r}}{\partial r}+c_{11} \frac{u_{r}}{r}+c_{13} \frac{\partial u_{z}}{\partial z}-\alpha_{h} p \\
& \sigma_{z z}=c_{13}\left(\frac{\partial u_{r}}{\partial r}+\frac{u_{r}}{r}\right)+c_{33} \frac{\partial u_{z}}{\partial z}-\alpha_{v} p \\
& \sigma_{r z}=c_{44}\left(\frac{\partial u_{z}}{\partial r}+\frac{\partial u_{r}}{\partial z}\right) \\
& p=-M \alpha_{h}\left(\frac{\partial u_{r}}{\partial r}+\frac{u_{r}}{r}\right) \\
& -M\left(\alpha_{v} \frac{\partial u_{z}}{\partial z}+\frac{\partial w_{r}}{\partial r}+\frac{w_{r}}{r}+\frac{\partial w_{z}}{\partial z}\right)
\end{aligned}
$$

where $\sigma_{r r}, \sigma_{\theta \theta}, \sigma_{z z}$ and $\sigma_{r z}$ denote the total stress components of the bulk material; $u_{i}$ and $w_{i}$ denote the displacement of the solid matrix and fluid displacement relative to the solid matrix respectively in the $i$-direction $(i=r, z) ; p$ is the excess pore fluid pressure (suction is considered negative); $c_{11}, c_{12}, c_{13}, c_{33}$ and $c_{44}$ are transversely isotropic elastic moduli ; and $\alpha_{i}(i=h, v)$ and $M$ are Biot's parameters accounting for compressibility of the two-phased material. In this paper, the variables $h$ and $v$ are used in the subscript of material parameters to define their properties either in the plane of isotropy $(i=h)$ or in the perpendicular direction to the isotropic plane $(i=v)$.

The relationship of Young's modulus, Poisson's ratio, and shear modulus with the elastic moduli, $c_{11}, c_{12}, c_{13}$, $c_{33}$ and $c_{44}$, can be expressed as follows:

$$
\begin{aligned}
& c_{11}=\frac{E_{h}\left(E_{v}-E_{h} v_{v h}^{2}\right)}{\left(1+v_{h}\right)\left(E_{v}-E_{v} v_{h}-2 E_{h} v_{v h}^{2}\right)} \\
& c_{12}=\frac{E_{h}\left(E_{v} v_{h}+E_{h} v_{v h}^{2}\right)}{\left(1+v_{h}\right)\left(E_{v}-E_{v} v_{h}-2 E_{h} v_{v h}^{2}\right)} \\
& c_{13}=\frac{E_{h} E_{v} v_{v h}}{E_{v}-E_{v} v_{h}-2 E_{h} v_{v h}^{2}} \\
& c_{33}=\frac{E_{v}^{2}\left(1-v_{v h}\right)}{E_{v}-E_{v} v_{h}-2 E_{h} v_{v h}^{2}} \\
& c_{44}=G
\end{aligned}
$$

where $E_{h}$ is the drained Young's modulus in the plane of isotropy; $E_{v}$ is the drained Young's modulus in the perpendicular direction to the isotropic plane; and $G$ is the shear modulus. In addition, $v_{h}$ is the drained Poisson's ratio characterizing the transverse strain reduction in the plane of isotropy due to tensile stress in the same plane; and $v_{v h}$ is the drained Poisson's ratio corresponding to the transverse strain reduction in the plane of isotropy due to tensile stress normal to it.

In this study, the motion is assumed to be timeharmonic with the factor of $e^{i \omega t}$, where $\omega$ is the frequency of the motion, and $i=\sqrt{-1}$. The term $e^{i \omega t}$ is suppressed hereafter from all equations and expressions for brevity.

The Hankel integral transform with respect to the radial coordinates is employed to solve for the general solution. The Hankel transform and its inverse transform can be expressed respectively as [19],

$$
\begin{aligned}
& \bar{f}(\xi, z)=\int_{0}^{\infty} f(r, z) J_{n}(\xi r) r d r \\
& f(r, z)=\int_{0}^{\infty} f(\xi, z) J_{n}(\xi r) \xi d \xi
\end{aligned}
$$

where $J_{n}$ denotes the Bessel function of the first kind of order $n$, and $\xi$ is the Hankel transform parameter.

It can be shown that the zero-order Hankel transforms of $u_{z}, w_{z}, \sigma_{z z}$, and $p$, and the first-order Hankel transforms of $u_{r}$ and $\sigma_{r z}$ for the case of axisymmetric loading applied on the surface of a half-space (see Fig. 1) can be expressed as, 


$$
\begin{aligned}
& \bar{u}_{r}=\chi_{1} A_{1} e^{-\lambda_{1} z}+\chi_{2} A_{2} e^{-\lambda_{2} z}+\chi_{3} A_{3} e^{-\lambda_{3} z} \\
& \bar{u}_{z}=\psi_{1} A_{1} e^{-\lambda_{1} z}+\psi_{2} A_{2} e^{-\lambda_{2} z}+\psi_{3} A_{3} e^{-\lambda_{3} z} \\
& \bar{p}=\zeta_{1} A_{1} e^{-\lambda_{1} z}+\zeta_{2} A_{2} e^{-\lambda_{2} z}+\zeta_{3} A_{3} e^{-\lambda_{3} z} \\
& \bar{w}_{z}=\varpi_{1} A_{1} e^{-\lambda_{1} z}+\varpi_{2} A_{2} e^{-\lambda_{2} z}+\varpi_{3} A_{3} e^{-\lambda_{3} z} \\
& \bar{\sigma}_{r z}=\varphi_{1} A_{1} e^{-\lambda_{1} z}+\varphi_{2} A_{2} e^{-\lambda_{2} z}+\varphi_{3} A_{3} e^{-\lambda_{3} z} \\
& \bar{\sigma}_{z z}=\eta_{1} A_{1} e^{-\lambda_{1} z}+\eta_{2} A_{2} e^{-\lambda_{2} z}+\eta_{3} A_{3} e^{-\lambda_{3} z}
\end{aligned}
$$

where $A_{1}, A_{2}$ and $A_{3}$ are the arbitrary functions to be determined from appropriate boundary conditions. In addition, $\lambda_{1}, \lambda_{2}$ and $\lambda_{3}$ are the dimensionless complex wave numbers associated with the three body waves, in which,

$$
\begin{aligned}
& \lambda_{1}=\sqrt{\Omega_{3}-\frac{\Omega_{1}}{3 \Omega_{3}}-\frac{\gamma_{2}}{3 \gamma_{1}}} ; \\
& \lambda_{2}=\sqrt{\Delta \Omega_{3}-\frac{\Omega_{1}}{3 \Delta \Omega_{3}}-\frac{\gamma_{2}}{3 \gamma_{1}}} ; \\
& \lambda_{3}=\sqrt{\Delta^{2} \Omega_{3}-\frac{\Omega_{1}}{3 \Delta^{2} \Omega_{3}}-\frac{\gamma_{2}}{3 \gamma_{1}}}
\end{aligned}
$$

Other parameters appearing in Eqs. (4) and (5) are explicitly shown in the Appendix.

To determine the influence functions corresponding to the vertical loading on the surface of the half-space, a boundary value problem has to be solved. For the case of uniformly distributed vertical loading of unit intensity applied over an annular area, the boundary conditions are given as follows:

$$
\begin{aligned}
& \bar{\sigma}_{r z}(\xi, 0)=0 \\
& \bar{\sigma}_{z z}(\xi, 0)=\bar{f}(\xi) \\
& \bar{p}(\xi, 0)=0
\end{aligned}
$$

where $\bar{f}(\xi)$ is the Hankel transform of the annular loading with the external and internal radii denoted by $r_{l o}$ and $r_{l i}$, respectively, and it is given by,

$$
\bar{f}(\xi)=\frac{1}{\xi}\left[r_{l o} J_{1}\left(\xi r_{l o}\right)-r_{l i} J_{1}\left(\xi r_{l i}\right)\right]
$$

The arbitrary functions $A_{1}, A_{2}$ and $A_{3}$ for the annular vertical loading on the surface of the half-space can be determined by solving the above boundary-value problem by substituting the relevant general solutions in Eq. (4) into the boundary conditions in Eq. (6). The vertical displacement in the Hankel transform domain is then obtained by substituting those arbitrary functions into Eq. (4b), and it is later used as the required influence function to solve the dynamic interaction problem between a circular plate and a transversely isotropic poroelastic half-space as shown in Fig. 1.

\section{Formulation of interaction problem}

Consider an elastic circular plate of radius $a$ subjected to axisymmetric vertical loading as shown in Fig. 1. The displacement of the plate is represented by $w(r)$ in the following form:

$w(r)=\sum_{n=0}^{N} \Delta_{n} r^{2 n}, \quad 0 \leq r \leq a$

where $\Delta_{n}(n=0,1, \ldots, N)$ denotes a set of generalized coordinates.

The strain and kinetic energies of the plate denoted by $U_{p}$ and $V_{p}$, respectively, can be expressed as [18]:

$$
\begin{gathered}
U_{p}=\frac{1}{2} \int_{0}^{a} 2 \pi D\left[\left(\frac{d^{2} w}{d r^{2}}+\frac{1}{r} \frac{d w}{d r}\right)^{2}\right. \\
\left.-\frac{2\left(1-v_{p}\right)}{r} \frac{d w}{d r} \frac{d^{2} w}{d r^{2}}\right] r d r \\
V_{p}=\frac{1}{2} h_{p} \int_{0}^{a} 2 \pi \rho_{p}[w(r)]^{2} r d r
\end{gathered}
$$

where

$$
D=\frac{E_{p} h_{p}^{3}}{12\left(1-v_{p}^{2}\right)}
$$

In the above equations, $h_{p}$ is thickness of the plate; and $E_{p}, v_{p}$, and $\rho_{p}$ are Young's modulus, Poisson's ratio, and the mass density of the plate material respectively.

A fully permeable circular plate under axisymmetric vertical loading is resisted by the normal contact traction acting on bottom surface of the plate. Let $S$ denote the circular contact area between the plate and the supporting medium. The normal traction can be represented by a traction field $T_{z}(r)$ acting on the circular surface $S$. The strain energy $U_{h}$ of the transversely isotropic poroelastic half-space can be expressed in the following form:

$U_{h}=\frac{1}{2} \int_{0}^{a} 2 \pi T_{z}(r) w(r) r d r$

In addition, the potential energy due to a vertical load, $q(r)$, as shown in Fig. 1 is written as,

$W=-\int_{0}^{a} 2 \pi q(r) w(r) r d r$ 
The discretization technique based on Zeng and Rajapakse [4] is employed to study this interaction problem. The circular contact area $S$ is discretized into a number of $N e$ annular elements. It is assumed that $T_{z}(r)$ is constant within each annular element. The unknown contact traction $T_{z}(r)$ is then evaluated by solving a flexibility equation based on the influence function as described in the previous section. The Lagrangian function $L$ of the plate given by Washizu [20] is defined as follows:

$$
L=V_{p}-U_{p}-U_{h}+W
$$

The generalized coordinates $\Delta_{n}(n=0,1, \ldots, N)$ are determined from the following equation:

$$
\frac{d}{d t}\left(\frac{\partial L}{\partial \Delta_{n}}\right)-\frac{\partial L}{\partial \Delta_{n}}=0, \quad n=0,1, \ldots, N
$$

The solution of a linear simultaneous equation system given by Eq. (12) yields the solution of the generalized coordinates $\Delta_{n}(n=0,1, \ldots, N)$ for a given plate-halfspace system. The deflection of the circular plate can then be obtained by substituting the generalized coordinates into Eq. (7).

\section{Numerical results and discussion}

In this study, a globally adaptive numerical quadrature scheme [21] with a 21-point Gauss-Kronrod rule is applied to the semi-infinite integral with respect to $\xi$, given by Eq. 3(b), to determine the required influence functions used for this interaction problem. In all numerical results presented hereafter, a non-dimensional relative flexibility parameter defined as $\gamma=G a^{3} / D$, and a non-dimensional frequency defined as $\delta=\omega a \sqrt{\rho / G}$ are used, in which $\rho$ is the mass density of the bulk material. In addition, a non-dimensional deflection of the circular plate is given by $w^{*}(r)=G w(r) / a q_{0}$ where $q_{0}$ is the magnitude of uniformly distributed loading acting on the circular plate. Other non-dimensional material parameters are defined as: $E_{h}^{*}=E_{h} / G ; \quad E_{v}^{*}=E_{v} / G$; $M^{*}=M / G ; \quad \rho_{f}^{*}=\rho_{f} / \rho ; \quad m_{h}{ }^{*}=m_{h} / \rho ; \quad m_{v}{ }^{*}=m_{v} / \rho ;$ $b_{h}{ }^{*}=b_{h} a / \sqrt{\rho G}$; and $b_{v}{ }^{*}=b_{v} a / \sqrt{\rho G}$, where $\rho_{f}$ is the mass density of the pore fluid; $m_{v}$ and $m_{h}$ are the densitylike parameters that depend on $\rho_{f}$ and the geometry of the pores; $b_{v}$ and $b_{h}$ are the parameters accounting for the internal friction due to the relative motion between the solid matrix and the pore fluid.

The accuracy of the present solution scheme is confirmed by comparing with existing solutions. Fig. 2 presents the comparison of non-dimensional deflections between the present solution and the solution given by Senjuntichai and Sapsathiarn [11] for the case of a circular plate, subjected to a uniformly distributed loading of constant magnitude $q_{0}$, resting on an isotropic poroelastic half-space. The material properties employed in the present solution are as follows: $\gamma=100 ; E_{h}{ }^{*}=E_{h}{ }^{*}$ $=2.6 ; v_{h}=v_{v h}=0.3 ; \alpha_{h}=\alpha_{h}=0.95 ; \rho_{f}{ }^{*}=0.53 ; m_{h}{ }^{*}=m_{h}$ $=1.1 ; M^{*}=12.2 ;$ and $b_{h}{ }^{*}=b_{h}{ }^{*}=2.3$. In addition, a smooth contract surface with fully permeable condition is assumed. Convergence of the proposed solution scheme was studied with respect to the number of annular elements, $\mathrm{Ne}$, and a set of generalized coordinates, $N$, employed in the proposed solution scheme. It can be clearly seen from Fig. 2 that the present solution, with $N e=32$ and $N=8$, agrees very closely with the existing solution [11] for both real and imaginary parts of the displacement at the center of the circular plate $(r=0)$.

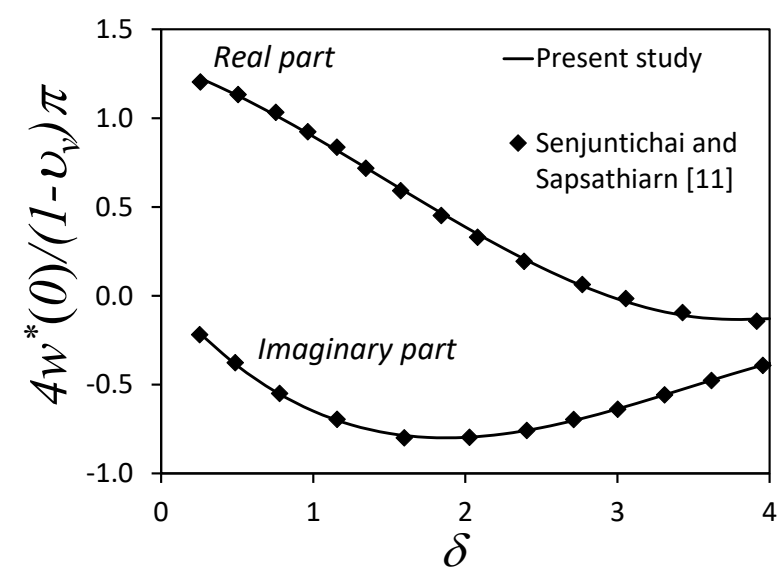

Fig. 2. Comparison with existing solutions for isotropic poroelastic half-space

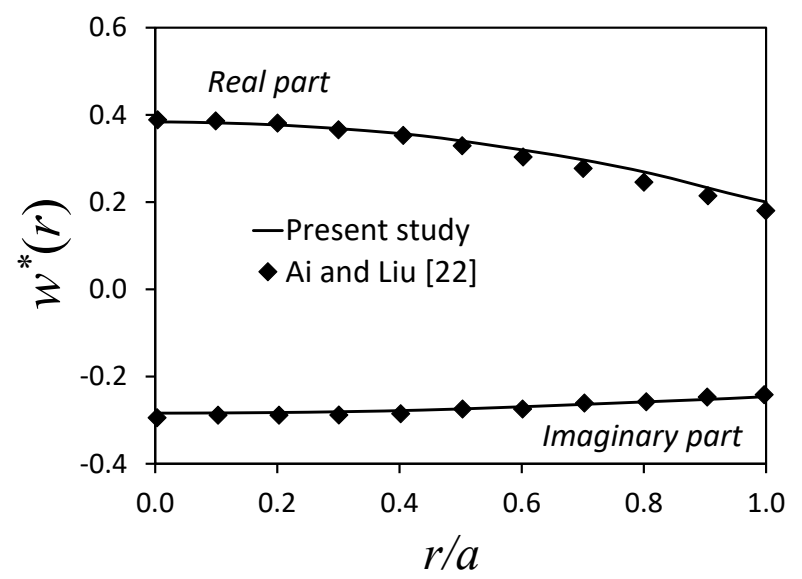

Fig. 3. Comparison with existing solutions for transversely isotropic elastic half-space

The accuracy of the present study is also examined in Fig. 3 by comparing with displacement profiles given by $\mathrm{Ai}$ and Liu [22] who studied the case of a circular plate, subjected to a uniformly distributed loading of constant magnitude $q_{0}$, resting on a transversely isotropic halfspace. The material properties employed in the present solution are as follows: $\delta=1 ; \gamma=11.25 ;{E_{h}}^{*}=2.5 ;{E_{h}}^{*}=$ 
7.5; $v_{h}=v_{v h}=0.3$ (other parameters are set to be negligibly small). It can be observed from Fig. 3 that a very good agreement between the presented result and that proposed by Ai and Liu [22] is obtained for both real and imaginary parts of $w^{*}(0)$.

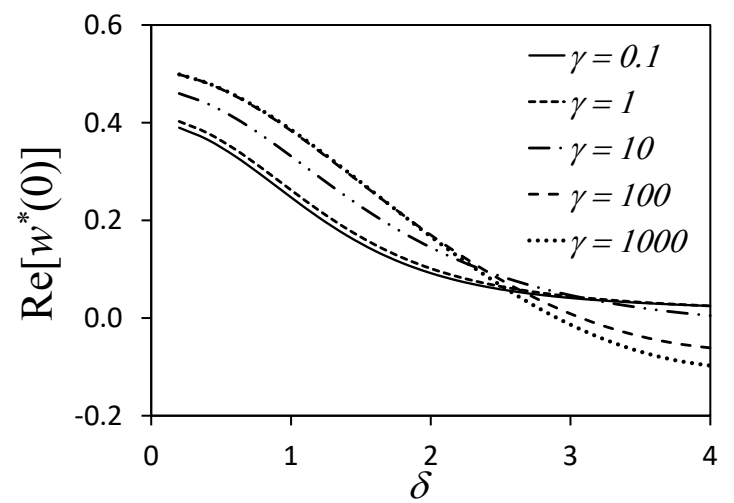

(a)

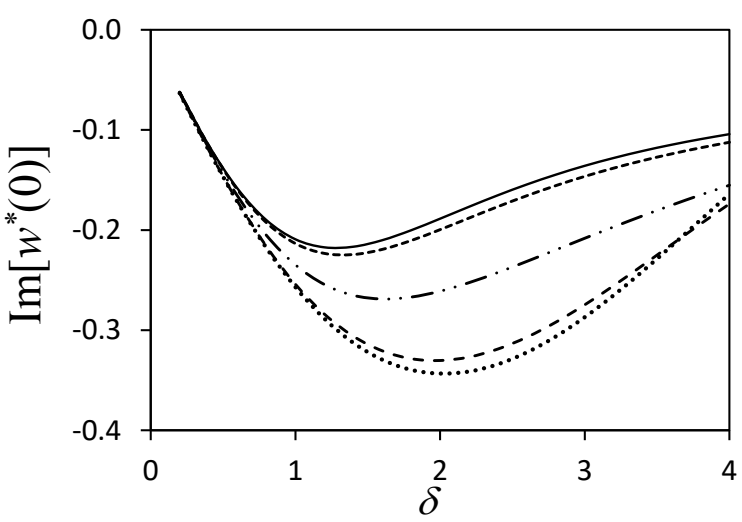

(b)

Fig. 4. Influence of relative flexibility on non-dimensional central deflection: (a) real part; (b) imaginary part

Next, vertical vibrations of a uniformly loaded circular plate resting on a transversely isotropic poroelastic half-space are shown in Figs. 4 and 5. The material properties employed in the presented results are defined as follows: $E_{h}{ }^{*}=2.5 ; E_{h}{ }^{*}=5 ; v_{h}=v_{v h}=0.3 ; \alpha_{h}$ $=\alpha_{h}=0.95 ; \rho_{f}{ }^{*}=0.5 ; m_{h}{ }^{*}=m_{h}=1.1 ; M^{*}=12.5 ;$ and $b_{h}{ }^{*}$ $=b_{h}{ }^{*}=2.5$. Fig. 4 shows both real and imaginary parts of non-dimensional deflection at the center of a circular plate for the non-dimensional frequency range of $\delta=$ 0.2-4.0. In addition, solutions are presented for different values of relative flexibility, i.e., $\gamma=0.1,1,10,100$ and 1000. It can be seen from the figure that the displacement of the plate depends significantly on both $\gamma$ and $\delta$. The magnitude of the displacements decreases with decreasing the relative flexibility for both real and imaginary parts of $w^{*}(0)$, except for $\delta=2.5-4.0$, where the increase of $w^{*}(0)$ with decreasing $\gamma$ is noted.
The influence of the relative flexibility on the deflection profiles of the circular plate is shown in Fig. 5 for $\delta=1$. It can be seen that the relative flexibility has a strong influence on the deflection profiles of the circular plate in the range $\gamma=1-100$. In addition, it is found that the plate behaves as a rigid disk when $\gamma$ is smaller than 0.1 . This implies that a flexible plate can be modelled as a rigid plate in this interaction problem when $\gamma \leq 0.1$.

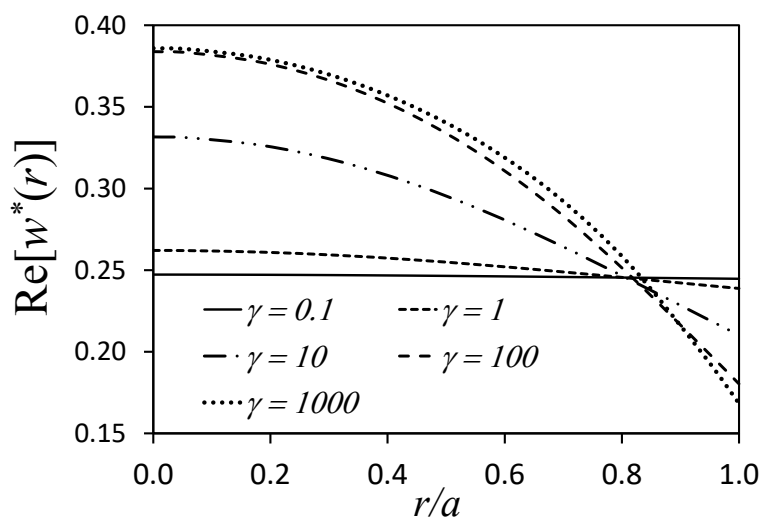

(a)

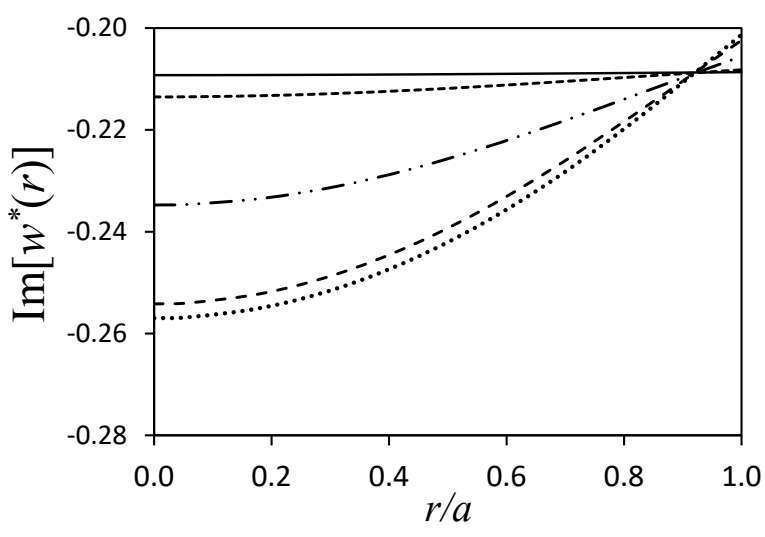

(b)

Fig. 5. Influence of relative flexibility on non-dimensional deflection profiles with $\delta=1$ : (a) real part; (b) imaginary part

\section{Conclusion}

This paper presents dynamic interaction between an elastic circular plate under vertical loading and a transversely isotropic poroelastic half-space. The interaction problem is investigated based on a variational principle together with appropriate influence function and discretization technique. The proposed scheme is verified with existing solutions for both isotropic poroelastic and transversely isotropic media. Selected numerical results are presented to demonstrate the influence of relative flexibility on the deflection of a circular plate. It is found that the influence of relative flexibility is significant when $\gamma=1-100$. Both real and 
imaginary parts of the deflection decrease with decreasing $\gamma$, and the maximum deflection is observed when $\gamma=1000$. It is also found that a circular plate with a small value of $\gamma$, i.e. $\gamma \leq 0.1$, can be considered as a rigid plate in the analysis of vertical vibrations of circular plate under axisymmetric vertical loading.

\section{Appendix}

The parameters in Eqs. (4) and (5) are given as follows:

$$
\begin{aligned}
& \chi_{j}=\xi \lambda_{j}\left[s_{4}\left(s_{3}+\beta_{v} \lambda_{j}^{2}\right)-s_{5} s_{6}\right] \\
& \psi_{j}=-\left(s_{3}+\beta_{v} \lambda_{j}^{2}\right)\left(s_{1}+c_{44} \lambda_{j}^{2}\right)+s_{6}^{2} \xi^{2} \\
& \zeta_{j}=\lambda_{j}\left[s_{5}\left(s_{1}+c_{44} \lambda_{j}^{2}\right)-s_{4} s_{6} \xi^{2}\right] \\
& \varpi_{j}=\left(\lambda_{j} \zeta_{j}-\rho_{f} \omega^{2} \psi_{j}\right) \beta_{v} \\
& \varphi_{j}=c_{44}\left(\lambda_{j} \chi_{j}-\xi \psi_{j}\right) \\
& \eta_{i}=\xi c_{13} \chi_{j}+c_{33} \lambda_{j} \psi_{j}-\alpha_{\nu} \zeta_{j} \\
& \Omega_{1}=-\frac{\gamma_{2}^{2}}{3 \gamma_{1}^{2}}+\frac{\gamma_{3}}{\gamma_{1}} ; \Omega_{2}=\frac{2 \gamma_{2}^{3}}{27 \gamma_{1}^{3}}-\frac{3 \gamma_{2} \gamma_{3}}{9 \gamma_{1}^{2}}+\frac{\gamma_{4}}{\gamma_{1}} ; \\
& \Omega_{3}=\left(-\frac{1}{2} \Omega_{2}+\frac{1}{2} \sqrt{\Omega_{2}^{2}+\frac{4 \Omega_{1}^{3}}{27}}\right)^{1 / 3} \text {; } \\
& \Delta=-\frac{1-\sqrt{3} i}{2} \\
& \gamma_{1}=c_{33} c_{44} \beta_{v} \\
& \gamma_{2}=\left(s_{2} c_{44}+s_{1} c_{33}+s_{4}^{2} \xi^{2}\right) \beta_{v}+\left(s_{3} c_{33}+s_{5}^{2}\right) c_{44} \text {; } \\
& \gamma_{3}=\left(s_{2} \beta_{v}+s_{5}^{2}\right) s_{1}+\left(s_{2} c_{44}+s_{1} c_{33}+s_{4}^{2} \xi^{2}\right) s_{3} \\
& -\left(2 s_{4} s_{5} s_{6}+s_{6}^{2} c_{33}\right) \xi^{2} ; \gamma_{4}=\left(s_{1} s_{3}-s_{6}^{2} \xi^{2}\right) s_{2} \\
& S_{1}=-\xi^{2} c_{11}+\omega^{2} \rho-\omega^{4} \rho_{f}^{2} \beta_{h} ; \\
& s_{2}=-\xi^{2} c_{44}+\omega^{2} \rho-\omega^{4} \rho_{f}^{2} \beta_{v} \text {; } \\
& s_{3}=-\xi^{2} \beta_{h}+\frac{1}{M} ; s_{4}=-\left(c_{13}+c_{44}\right) \\
& s_{5}=\alpha_{v}-\omega^{2} \rho_{f} \beta_{v} ; s_{6}=\alpha_{h}-\omega^{2} \rho_{f} \beta_{h} \\
& \beta_{j}=\frac{1}{\left(m_{j} \omega^{2}-i b_{j} \omega\right)} \quad(j=h, v)
\end{aligned}
$$

This work was supported by the Thailand Research Fund (grant number PHD/0064/2558).

\section{References}

1. M.A. Biot, J. Acoust. Soc. Am. 28, 168-179 (1956)

2. M.K. Kassir, J. Xu, Int. J. Solid Struct. 24, 915-936 (1988)

3. B. Jin, H. Liu, Soil. Dyn. Earthquake Eng. 18, 437443 (1999)

4. X. Zeng, R.K.N.D. Rajapakse, Int. J. Numer. Analyt. Meth. Geomech. 23, 2075-2095 (1999)

5. Z.Y. Ai, C.L. Liu, J.P. Jiang, Meccanica 51, 18871895 (2016)

6. M.R. Halpern, P. Christiano, Earthquake Eng. Struct. Dyn. 14, 439-454 (1986)

7. T. Senjuntichai, S. Keawsawasvong, R. Plangmal, Mar. Georesour. Geotec. (to be published)

8. T. Senjuntichai, S. Keawsawasvong, R. Plangmal, Comput. Geotech. 100, 121-134 (2018)

9. S. Keawsawasvong, T. Senjuntichai, IOP Conference Series: Materials Science and Engineering, 269, 012047 (2017)

10. T. Senjuntichai, W. Kaewjuea, J. Mech. Mater. Struct. 3(10), 1885-1901 (2008)

11. T. Senjuntichai, Y. Sapsathiarn, The Eighth East Asia-Pacific Conference on Structural Engineering and Construction, Nanyang Technological University (2001)

12. M.A. Biot, J. Appl. Phys. 26, 182-185 (1955)

13. M.A. Biot, J. Appl. Phys. 33, 1482-1498 (1962)

14. A.H.D. Cheng, Int. J. Rock Mech. Min., 34(2), 199205 (1997)

15. R. Kumar, N.R. Garg, A. Miglani, J. Sound Vib., 261, 697-714 (2003)

16. K. Sahebkar, M. Eskandari-Ghadi, J. Hydrol., 537, 61-73 (2016)

17. A. Pooladi, M. Rahimian, R.Y.S. Pak, Appl. Math. Model., 50, 177-199 (2017)

18. S.P. Timoshenko, S. Woinowsky-Krieger, Theory of plates and shells (McGraw-Hill, 1959)

19. I.N. Sneddon, Fourier Transforms (McGraw-Hill, 1951)

20. K. Washizu, Variational methods in elasticity and plasticity, $2^{\text {nd }} E d$ (Pergamon, 1982)

21. R. Piessens, E. Doncker-Kapenga, C.W. Uberhuber, D.K. Kahaner, QUADPACK, A subroutine package for automatic integration (Springer, 1983)

22. Z.Y. Ai, C.L. Liu, Soil. Dyn. Earthquake Eng. 67, 257-263 (2014) 